\title{
Collaborative Semantic Points of Interests
}

\author{
Max Braun, Ansgar Scherp, and Steffen Staab \\ University of Koblenz-Landau, Germany \\ \{maxbraun, scherp, staab\}@uni-koblenz.de \\ http://isweb.uni-koblenz.de
}

\begin{abstract}
The novel mobile application csxPOI (short for: collaborative, semantic, and context-aware points-of-interest) enables its users to collaboratively create, share, and modify semantic points of interest (POI). Semantic POIs describe geographic places with explicit semantic properties of a collaboratively created ontology. As the ontology includes multiple subclassifications and instantiations and as it links to DBpedia, the richness of annotation goes far beyond mere textual annotations such as tags. Users can search for POIs through the subclass hierarchy of the collaboratively created ontology. For example, a POI annotated as bakery can be found through the search string shop as it is a superclass of bakery. Data mining techniques are employed to cluster and thus improve the quality of the collaboratively created POIs.
\end{abstract}

\section{Introduction}

Mobile devices with permanent Internet connectivity are turning the vision of ubiquitous computing into reality [1. Applications running on such devices are among others aware of the user's geographic location and can adapt their behavior and content accordingly. Points of interests (POIs) provide useful information about specific geographic places. Whether users are able to quickly find the POIs they are interested in, depends among others on the quality of the POIs' annotations. Unstructured textual descriptions of POIs and folksonomic tags are a good starting point. However, the relation between the POIs remains hidden in the data and is hard to extract and understand for the machine. Other (mobile) systems such as [2,3] provide a semantic representation of POIs but do not provide for a collaborative creation and modification of semantic POIs and an underlying ontology of POI categories.

In order to understand semantic POIs and their relations, we have developed the mobile application csxPOI (short for collaborative, semantic, and contextaware points of interests) to collaboratively create, share, and modify semantic POIs. While working with the POIs, the users collaboratively modify and improve an ontology of POI categories underlying to the application. Such usercontributed POIs gathered from a large group of people is likely to include duplicate POIs with similar but unequal annotations and slightly varying locations for the same physical places. Thus, a revision engine applies data mining techniques for POI clustering.

L. Aroyo et al. (Eds.): ESWC 2010, Part II, LNCS 6089, pp. 365-369, 2010.

(C) Springer-Verlag Berlin Heidelberg 2010 


\section{Collaborative Ontology for Points of Interests}

We have created a set of ontologies for modeling the structure of POIs, content of POIs, user accounts, and most notably the history of all collaborative user activities. The base ontology defines the fundamental concepts such as POI, contribution, user, and their relationships. The vocabulary ontology defines POI categories such as monument, park, and others. It also provides the properties, relationships, and interlinks of the POIs. The vocabulary ontology is open to direct collaborative modification by users of the csxPOI application. The POI ontology comprises all instances of POIs and their associated metadata. As such, the ontology directly depends on the categories defined in the vocabulary ontology. Users of the csxPOI application primarily interact with this ontology during POI creation and POI retrieval. The collaboration ontology represents any kind of collective activity in the csxPOI application and are called contributions. A contribution is either the creation, modification, or deletion of a POI as well as the creation, modification, and deletion of a POI category in the vocabulary ontology. The collaboration ontology directly depends on the previous three ontologies. Finally, the user ontology contains all instances of users of the csxPOI application and their respective account information. As the first users of our csxPOI application have not yet created their own POI categories, there is an initial vocabulary ontology extracted automatically from LinkedGeoData [4].

\section{The csxPOI Application}

The default screen of the csxPOI application is shown in Figure 1(a). Once registered, users can Create POIs at the position centered on the map. Subsequently, a dialog containing a text field for the POI's name is shown. In addition, semantic categories of the POI can be entered. Figure 1(b) shows a sample POI created for the Monument to Kaiser Wilhelm I in Koblenz, Germany. The screenshot shows the POI with its German name Kaiser-Wilhelm-I.-Denkmal and a list of the two categories named attraction and monument. Categories are added by entering their name in the corresponding text field which features a semantic auto-completion function. A sample usage of the semantic auto-completion can be seen in Figure 1(b) where the categories beginning with mo are shown. Each entry corresponds to a category from the collaborative POI ontology introduced in Section 2 with all its relations and interlinks. The user can either select one of the suggested categories and annotate the POI by pressing the plus button or entering a new name, i.e., a category that is not included in the ontology. Entering a new category opens the ontology editor for further specification of it.

Users can Find POIs in the vicinity and display them on the map. Here, two options are possible: semantic search and presenting all POIs associated with the current user. A sample result for the semantic search bridge can be seen in Figure 1(d), where the POIs are marked with star symbols. The feature Show my POIs depicted in Figure 1(a) provides a list of all POIs a user created or modified. The details of a POI displayed on the map can be shown by tapping 


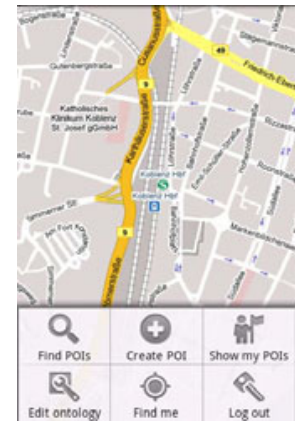

(a) Main menu

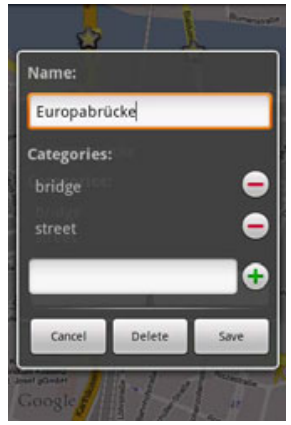

(e) Editing a POI

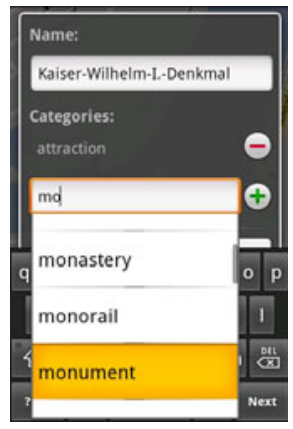

(b) Auto-completion

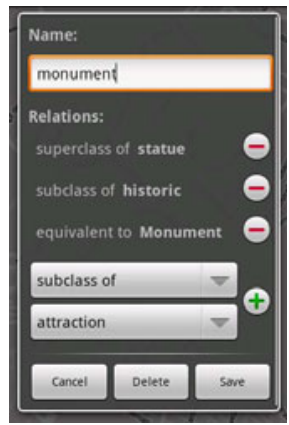

(f) Category editing

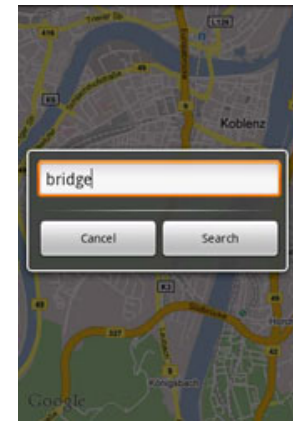

(c) Semantic search

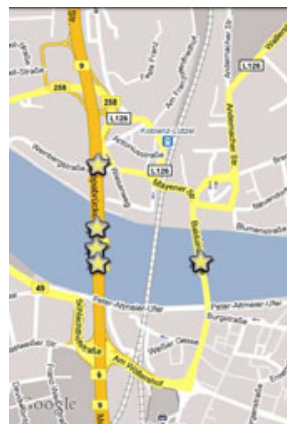

(g) Duplicate POIs

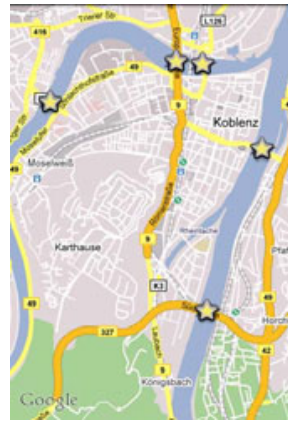

(d) Search results

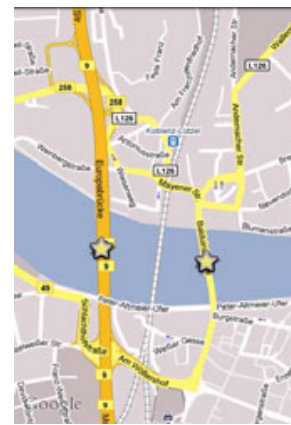

(h) Revised POIs

Fig. 1. Screenshots of our csxPOI application showing its different features

on it. It shows the name at the top and below a list of all categories the POI belongs to. For example, a POI named Europabrücke (in english: Europe bridge) with the two categories bridge and street is shown in Figure 1(e). The semantic annotation allows to search along the hierarchy of concepts a POI belongs to. For example, a POI that is annotated with the concept bakery can be found through the search term shop as the concept shop is a superclass of bakery.

The user can Edit the POI, when logged in. Figure 1(e) shows the POI editor for the previous example. The POI name can be edited in a text field at the top. Categories are added by entering their name in the text field at the bottom with support of semantic auto-completion and pressing the plus button. This may open the ontology editor if a category name is unknown. Removing categories is conducted via the minus button.

Besides creating and editing semantic POIs, the collaborative POI ontology can also be edited directly through the ontology editor. An example is shown in Figure 1(f) for the category monument. Users can edit the category's name in the text field. Below is a list of relations, each representing one triple with the edited category as the subject of the triple. The predicate is defined by the relation type. It can be one of subclass of, superclass of, and equivalent to. The former two correspond to the property rdfs:subClassOf and its inverse, the latter to owl:sameAs. The object of the triple is the target category. It is shown on the right hand side and can be any category from the vocabulary ontology. 
In addition, it can be a concept from an external resource such as DBpedia (http://dbpedia.org/). Relations are added and removed by choosing the relation type and category from the lower drop-down menus as shown in Figure 1(h) and pressing the plus button and minus button, respectively.

\section{Revision of Collaboratively Created POIs}

The csxPOI application provides a revision engine that clusters duplicate POIs with combinations of spatial, linguistic, and semantic similarity measures. The revision engine follows a two-step approach: In a first step, the spatial similarity is measured by calculating the distance between two POIs and mapping it to inverse values in $[0,1]$ with 1 indicating the identical location and 0 indicating maximal distance. The distance is based on the WGS 84 reference ellipsoid modeling the earth's surface [5]. Linguistic similarity is modeled by the JaroWinkler [6] string metric between the labels of two POIs. In the csxPOI ontology, POIs are instances of categories which themselves have subclass relations among each other. This taxonomic structure is utilized to assess a semantic similarity between POIs by comparing their relative position in the hierarchy. This is computed with the asymmetric similarity measure MDSM [7]. The different metrics are integrated with a weighted linear combination of their individual normalized values. In the second step, clusters of POIs are determined employing the DBSCAN algorithm [8. We choose the medoid as representative of the cluster. An owl:sameAs relation pointing to the medoid is then added to the members of the cluster. Figure $1(\mathrm{~g})$ shows a screenshot where five semantic POIs have been created. The four POIs on the left are all variations of the actual bridge and vary in location and the name given, which is Europabrücke (two times), Europa-Brücke, and Europabrucke. These four POIs are categorized as bridges as they instantiate the category bridge. But some additionally belong to the street category and others to pedestrian. The bridge can be used by both vehicles and pedestrians, which lead to this ambivalent categorization. The POI on the right has the name Balduinbrücke and also represents a bridge. After clustering, the four POIs on the left have been merged into one POI as shown in Figure 1(h)

\section{Related Work}

IYOUIT is one of today's most sophisticated mobile context-aware applications [2]. It features social relationships, location records, and weather conditions, which are stored in formal ontologies. DBpedia [3] is an effort to extract structured information from Wikipedia and publish it in Linked Data. DBpedia Mobile [9] is a mobile client to explore that data. It uses the client's GPS sensor and semantic datasets to display nearby places on a map. DBpedia Mobile's Linked Data browser provides the user with background information about discovered places. In addition, there are several other applications that allow for mobile creation and sharing of POIs such as Wikitude (http://www.wikitude.org/). Discussing all of those applications is beyond the scope of this paper. A nice application for mobile creation and sharing of 
multimedia documents is Haiku (http://haikus.tid.es) where users can leave comments and annotate the provided content. Unlike our approach, none of the existing approaches allow for the semantic annotation and collaborative creation of a network of semantic POIs and their respective categories.

\section{Conclusions}

We presented a novel mobile application csxPOI for collaboratively creating, sharing, and modifying semantic points of interest. Additionally, the vocabulary ontology comprising the POI categories used for the semantic annotation of POIs is itself a product of a collaborative process. As user-contributed POIs inherently introduce duplicate POIs, we provide a POI revision engine based on data mining techniques to improve the quality of the collaborative POI data set. To fill the cold start up situation, we investigate among others detecting events on the social media sharing platform Flickr and presenting images of those events. Further information and demo videos can be found at: http://west.uni-koblenz.de/Research/systeme/csxPOI

Acknowledgment. This research has been co-funded by the EU in FP7 in the WeKnowIt project (215453).

\section{References}

1. Weiser, M.: The Computer for the 21st Century. Scientific American 265(3) (1991)

2. Boehm, S., Koolwaaij, J., Luther, M., Souville, B., Wagner, M., Wibbels, M.: Introducing IYOUIT. In: Sheth, A.P., Staab, S., Dean, M., Paolucci, M., Maynard, D., Finin, T., Thirunarayan, K. (eds.) ISWC 2008. LNCS, vol. 5318, pp. 804-817. Springer, Heidelberg (2008)

3. Auer, S., Bizer, C., Kobilarov, G., Lehmann, J., Cyganiak, R., Ives, Z.: DBpedia: A Nucleus for a Web of Open Data. In: Aberer, K., Choi, K.-S., Noy, N., Allemang, D., Lee, K.-I., Nixon, L.J.B., Golbeck, J., Mika, P., Maynard, D., Mizoguchi, R., Schreiber, G., Cudré-Mauroux, P. (eds.) ASWC 2007 and ISWC 2007. LNCS, vol. 4825, pp. 722-735. Springer, Heidelberg (2007)

4. Auer, S., Lehmann, J., Hellmann, S.: Linkedgeodata: Adding a spatial dimension to the web of data. In: Bernstein, A., Karger, D.R., Heath, T., Feigenbaum, L., Maynard, D., Motta, E., Thirunarayan, K. (eds.) ISWC 2009. LNCS, vol. 5823, pp. 731-746. Springer, Heidelberg (2009)

5. National Imagery and Mapping Agency (NIMA): Department of Defence World Geodetic System 1984. NIMA Technical Report 8350.2 (2004)

6. Winkler, W.E.: The State of Record Linkage and Current Research Problems. R99/04, Statistics of Income Division, U.S. Census Bureau (1999)

7. Rodríguez, M.A., Egenhofer, M.J.: Comparing Geospatial Entity Classes: An Asymmetric and Context-Dependent Similarity Measure. Int. Journal of Geographical Information Science 18 (2004)

8. Ester, M., Kriegel, H.P., Sander, J., Xu, X.: A Density-Based Algorithm for Discovering Clusters in Large Spatial Databases with Noise. In: 2nd Int. Conf. on Knowledge Discovery and Data Mining (1996)

9. Becker, C., Bizer, C.: DBpedia Mobile: A Location-Enabled Linked Data Browser. In: 1st Int. Workshop on Linked Data on the Web (2008) 